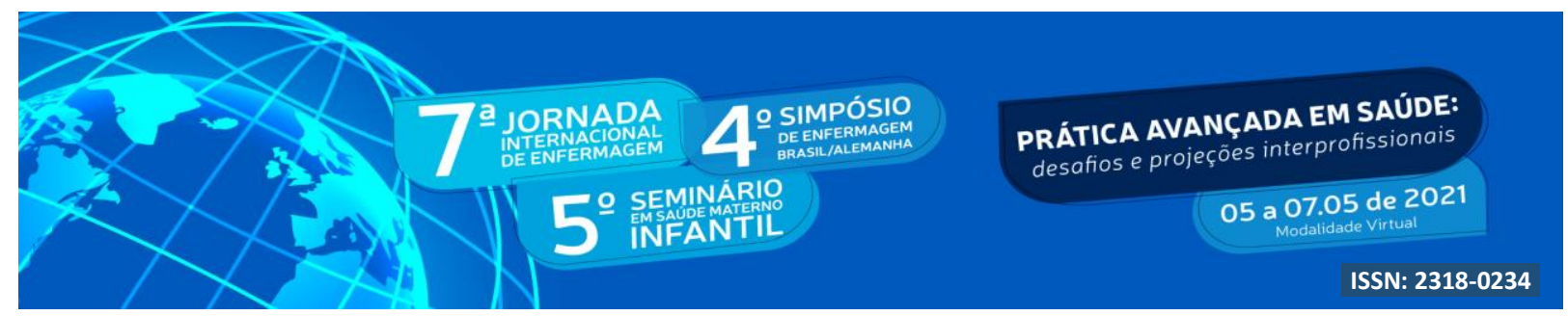

DOI: http://doi.org/10.48195/jie2021-155

\title{
PESQUISAS REALIZADAS NA ATENÇÃO PRIMÁRIA EM SAÚDE: PARA QUEM E COM QUEM? ${ }^{1}$
}

\section{Angélica Lucion Farinha²; Carolina Fernandes Rocha ${ }^{3}$; Carolina Felli Kubiça ${ }^{4}$; Daiany Donaduzzi $^{5}$; Fábio Mello da Rosa ${ }^{6}$; Juliana Silveira Colomé ${ }^{7}$}

\begin{abstract}
RESUMO
Esta pesquisa tem como objetivo caracterizar as pesquisas desenvolvidas na Atenção Primária em Saúde município de Santa Maria - RS, entre os anos de 2014 e 2020. Trata-se de uma pesquisa do tipo documental com abordagem quantitativa, desenvolvida no Núcleo de Educação Permanente em Saúde de Santa Maria e vinculada ao Programa de Educação pelo Trabalho para a Saúde/ PET-Saúde Interprofissionalidade. Foram analisados os projetos de pesquisa desenvolvidos nos últimos sete anos, considerando as seguintes variáveis: local de realização; participantes e instrumentos de coleta de dados utilizados. O estudo mostrou que as pesquisas realizadas na APS são em sua maioria realizadas nas Unidades Básicas de Saúde, tendo como participantes majoritoriamente os usuários e utilizando como instrumento de coleta a entrevista. A caracterização das pesquisas é importante, pois além de demostrar quais conteúdos e problemas de saúde estão sendo investigados, pode apresentar resultados que tragam beneficios aos serviços de saúde e usuários além de incentivar novos estudos.
\end{abstract}

Palavras-chave: Serviços de Integração Docente Assistencial; Sistema Único de Saúde; Pesquisas.

\section{ABSTRACT}

This research aims to characterize the research developed in Primary Health Care in the municipality of Santa Maria - RS, between the years 2014 and 2020. It is a documentary type research with a

\footnotetext{
${ }^{1}$ Documental, quantitativo. Conselho Nacional de Desenvolvimento Científico e Tecnológico.

${ }^{2}$ Bolsista de Iniciação Científica, PIBIC-CNPq. Estudante do Curso de Enfermagem. Universidade Franciscana UFN. E-mail: angelicaluciom2011@ @otmail.com

${ }^{3}$ Bolsista PET-Saúde Interprofissionalidade. Estudante do Curso de Enfermagem. Universidade Franciscana UFN.

${ }^{4}$ Bolsista PET- Saúde Interprofissionalidade. Estudante do Curso de Psicologia. Universidade Franciscana UFN.

${ }^{5}$ Doutoranda em Enfermagem. Superintendente da Atenção Básica de Santa Maria - RS. Preceptora do PETSaúde Interprofissionalidade da Universidade Franciscana - UFN.

${ }^{6}$ Mestre em Enfermagem. Coordenador do Núcleo de Educação Permanente em Saúde de Santa Maria - RS.

Coordenador do PET-Saúde Interprofissionalidade da Universidade Franciscana - UFN.

7 Doutora em Enfermagem. Docente e Coordenadora de Grupo do PET-Saúde Interprofissionalidade da Universidade Franciscana - UFN.
} 


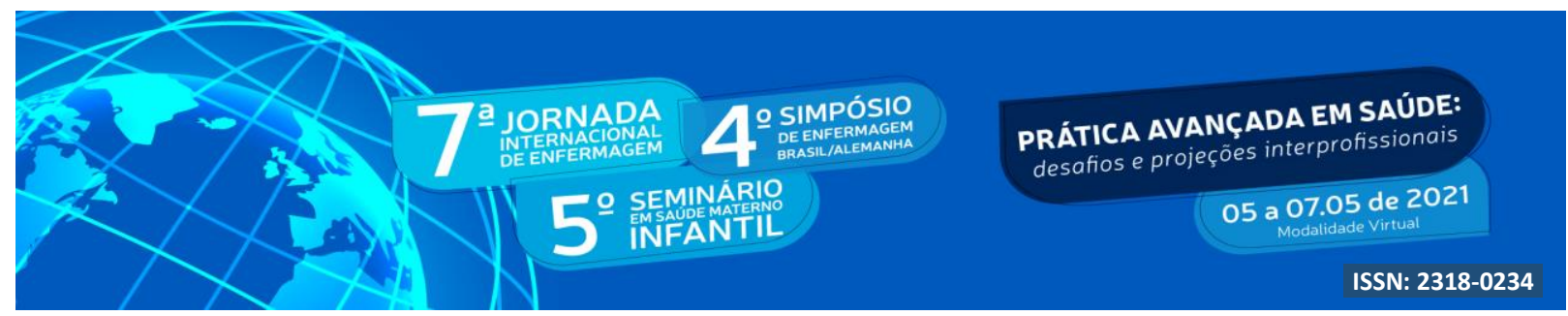

quantitative approach, developed at the Center for Permanent Education in Health Santa Maria and linked to the Education through Work Program for Health / PET-Saúde Interprofessionality. Research projects developed in the last seven years were analyzed, considering the following variables: place of performance; participants and data collection instruments used. The study showed that the researches carried out in PHC are mostly carried out in Basic Health Units, with the majority of users participating and using the interview as a collection instrument. The characterization of the research is important, because in addition to showing what content and health problems are being investigated, it can present results that bring benefits to health services and users, in addition to encouraging new studies.

Key Words: Teaching Assistance Integration Services; Health Unic System; Researches.

\section{INTRODUÇÃO}

A atenção primária a saúde (APS) é caracterizada por um conjunto de ações de saúde, no contexto individual e coletivo, que compreende ações de promoção, proteção da saúde, prevenção de agravos, diagnóstico, tratamento, reabilitação, redução de danos e a conservação da saúde que tem como objetivo desenvolver uma atenção integral que traga efeitos na situação de saúde e autonomia da população e nos determinantes e condicionantes de saúde das coletividades (BRASIL, 2017 a). As Unidades Básicas de Saúde (UBS), constituem um ambiente efetivo para a qualificação que busca a melhor organização dos serviços e uma maior oferta de ações a população. Para isso, é necessário profissionais com conhecimentos, habilidades e atitudes singulares, capacitados para o desenvolvimento de práticas de cuidado integral à saúde de forma reflexiva e construtiva, formando equipes de atenção básica à saúde (CAVALCANTI; OLIVEIRA NETO; SOUSA, 2015).

Para o cuidado em saúde ser realizado de forma integral é necessário, a resolutividade dos problemas sociais e de saúde da população o que exige que as diretrizes norteadoras das ações estejam orientadas por evidências científicas. Desta forma é importante tratar a pesquisa como uma considerável ferramenta para a geração de informações que facilitem a tomada de decisão gerando assim conhecimentos aplicáveis, capazes de possibilitar melhoras nos serviços da APS. Toda via, para isso é necessário estreitar o vínculo entre instituição e 


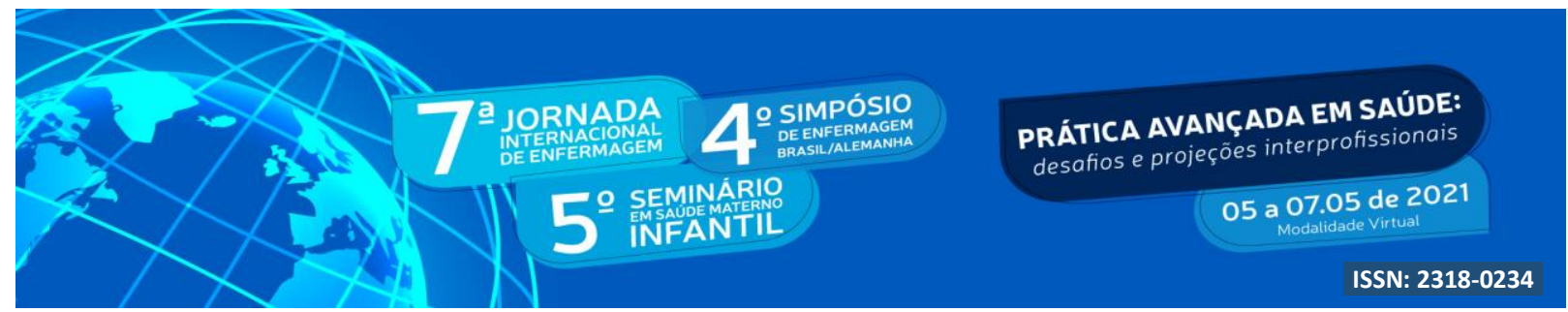

serviços de saúde, fortalecer a integração ensino serviço comunidade e também colaborando na aproximação de pesquisadores comprometidos com os temas prioritários e gestores sensibilizados à incorporação dos resultados dos estudos na formulação de políticas e nas intervenções em saúde, sobretudo, nos serviços de saúde do SUS (MORAIS, et al, 2018).

Afim de que os avanços sejam efetivos no Sistema Único de Saúde, e que o cuidado seja humanizado, interprofissional, interdisciplinar, em redes de atenção e pautado em princípios éticos é necessário a formação vivenciada por estudantes durante a graduação, pois estes serão os futuros profissionais que atuarão no cuidado da população. Conviver desde a graduação nas UBS mostra-se como uma possibilidade efetiva para a busca ativa do conhecimento, do autoaprendizado, do desenvolvimento da comunicação, da crítica e da reflexão. Além disso percebeu-se também que algumas das orientações das Ditreizes Curriculares Nacionais (DCN) estão sendo vivenciadas pelos acadêmicos nas UBS, emergi então a reflexão que os planejamentos que movem gestores e docentes a buscarem integrar ensino-serviço de saúde parecem encontrar acordo com o que é vivenciado pelos estudantes quando habitam a UBS (CODATO, et al, 2019).

A integração ensino serviço comunidade é um dos elementos que impulsiona, no Brasil, o movimento de mudanças na formação dos profissionais de saúde e constitui uma estratégia coletiva efetiva para a qualificação do SUS, visto que integra usuários, estudantes, professores e profissionais das equipes dos serviços de saúde. Dentre os objetivos primordiais, destaca-se a qualidade da atenção prestada ao usuário, a excelência da formação profissional e o desenvolvimento dos trabalhadores dos serviços (BRITO et al., 2017).

A integração ensino serviço comunidade no Brasil retrata relevante ferramenta para que as ações realizadas no SUS sejam efetivas, ao ligar e buscar aliança entre a formação profissional, oferecida pelos cursos de graduação, e os serviços de atenção à saúde ofertados pelo SUS. Para que o SUS seja efetivo, é necessário e fundamental investir na formação e desenvolvimento de seus servidores, que possuem papel de agentes de transformações necessárias para os avanços desejados. Na construção de habilidades e atitudes, as ações educativas devem ser compreendidas, para além do sentido clássico da aquisição de conhecimentos técnico-científicos, como um processo de formação de sujeitos críticos e reflexivos, de transformação da realidade e de criação de novas formas de gestão dos 


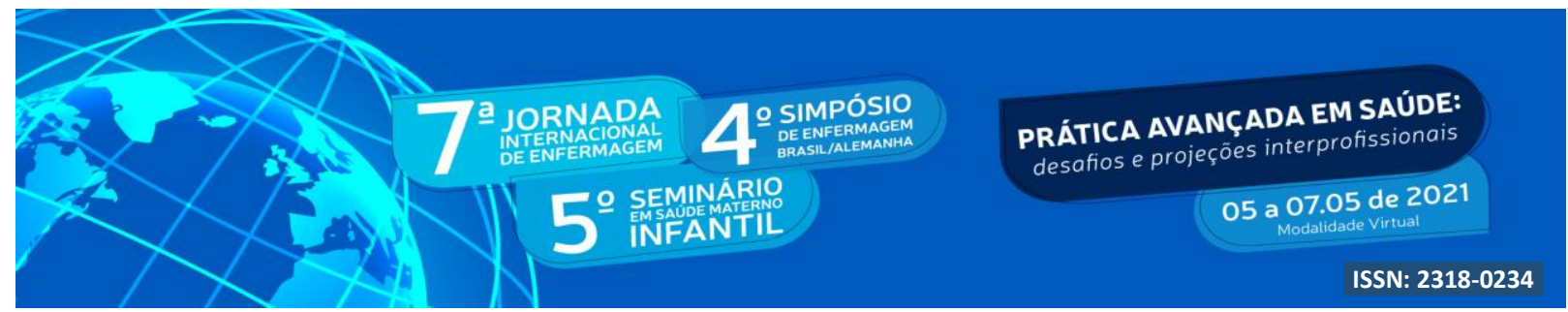

processos de trabalho. Ao integrar o mundo do trabalho ao mundo da educação, o ambiente de aprendizagem dos estudantes e trabalhadores configura-se no próprio espaço da atenção e gestão do SUS. Essa aproximação faz com que o aprendizado seja fundamentado na reflexão das práticas, ganhando sentido por estar relacionado à realidade do trabalho em saúde (BRASIL, 2017 b).

Vista disso o Núcleo de Educação Permanente em Saúde (NEPeS) é uma estratégia do Sistema Único de Saúde (SUS) para a formação e o desenvolvimento de trabalhadores para o setor, contemplando os aspectos da Educação Permanente em Saúde (EPS) e da integração ensino-serviço-comunidade, assim como o Pet Saúde também o faz. Segundo o Plano Municipal de Saúde de Santa Maria (PMSM/SMS, 2018, p.92), uma das metas do eixo norteador "Educação em Saúde" é a seguinte: "Garantir que 100\% dos projetos de pesquisa e extensão sejam realizados somente após aprovação do Nepes", desta forma torna-se possível armazenar esses dados e assim identificar o perfil das pesquisas da APS realizadas no munícipio de Santa Maria.

Dentre as estratégias efetivas para a integração ESC, destaca-se a criação dos programas Pró-Saúde e o PET-Saúde. Estes foram criados com vistas a introduzir a educação interprofissional nos cursos de graduação em saúde, bem como integração do ensino e serviço, de comum acordo com os preceptores e supervisores dos campos de atuação onde os estudantes estão inseridos. Tais estratégias têm o objetivo de fomentar para que a formação dos profissionais de saúde seja voltada para o cuidado integral, além de profissionais comprometidos em atender as necessidades com que poderão se deparar no serviço (CAMARA; GROSSEMAN; PINHO, 2015).

O PET-Saúde promove ações de integração ESC, com interação com a realidade, objetivando formar profissionais conscientes das necessidades que se apresentam nos serviços de saúde. Além de estimular os profissionais que já atuam nestes serviços a constantes capacitações e atualizações, reforçando a importância de serem produtores de conhecimentos e práticas, tornando-os capazes de atender às diferentes demandas da população atendida pelo SUS (BRASIL, 2012). O programa contribui com bolsas para tutores (professores), preceptores (profissionais dos serviços) e estudantes de graduação da área da saúde. 


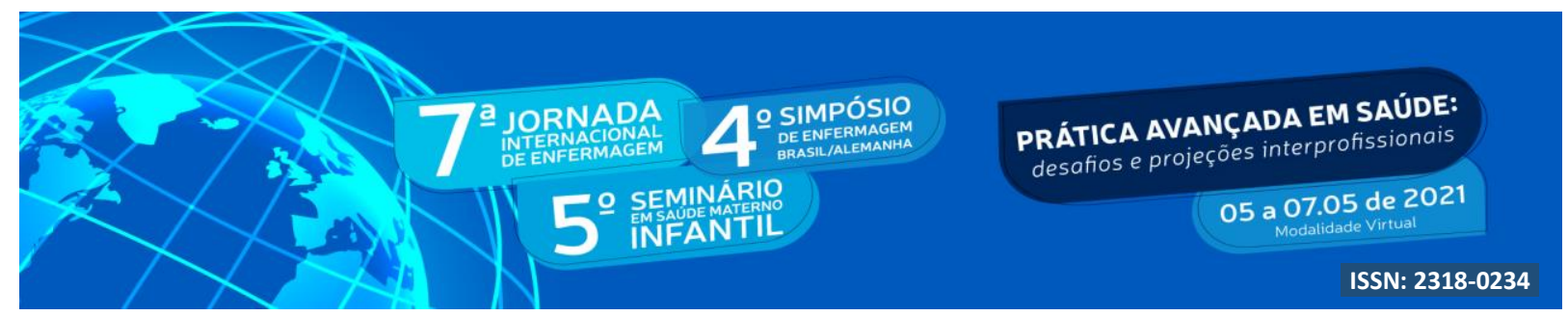

\section{OBJETIVO}

Caracterizar as pesquisas desenvolvidas na Atenção Primária em Saúde município de Santa Maria - RS, entre os anos de 2014 e 2020.

\section{METODOLOGIA}

A presente pesquisa trata-se de uma pesquisa do tipo documental com abordagem quantitativa, desenvolvida no âmbito do Programa de Educação pelo Trabalho para a Saúde Interprofissionalidade (Pet Saúde Interprofissionalidade). A análise documental, também conhecida como pesquisa documental, é caracterizada pela investigação de documentos de carater internos ou externos, é um método utilizado tanto para pesquisas quantitativas como qualitativas (ZANELLA, p.118, 2013).

As bolsistas iniciaram o levantamento de dados a partir do ano 2019 e devido às restrições impostas pela pandemia de COVID-19 no ano de 2020, a pesquisa no NEPeS teve fim em fevereiro de 2021. Desse modo, foram analisados os projetos de pesquisa desenvolvidos entre os anos de 2014 e 2020. O agrupamento dos dados foi realizado através de planilhas do programa Excel, contendo as informações dos referidos projetos de pesquisa. Foram analisados os projetos de pesquisa desenvolvidos nos últimos sete anos, considerando as seguintes variáveis: local de realização; participantes e instrumentos de coleta de dados utilizados.

Para tornar possível a análise dos dados, cada um destes tipos foi categorizado, tornando possível uma melhor visualização dos resultados da pesquisa. Dessa forma, a variável "local de realização" foi dividida em cinco categorias: Unidade Básica de Saúde (UBS); Estratégia de Saúde da Família (ESF); Mista (UBS e ESF); Rede de Atenção à Saúde (RAS) e Sistema de Gestão. A variável "participantes" foi organizada em dividido em sete categorias: Gestores; Usuários; Profissionais; Alunos; Professores (Programa Saúde na Escola-PSE); Documental/ Prontuário; Outros (não foi possível definir). Por sua vez, a variável "instrumento de coleta de dados" foi dividida em sete categorias: Questionário; Entrevista; Análise de documentos e/ou prontuário; Abordagem grupal; Análises 


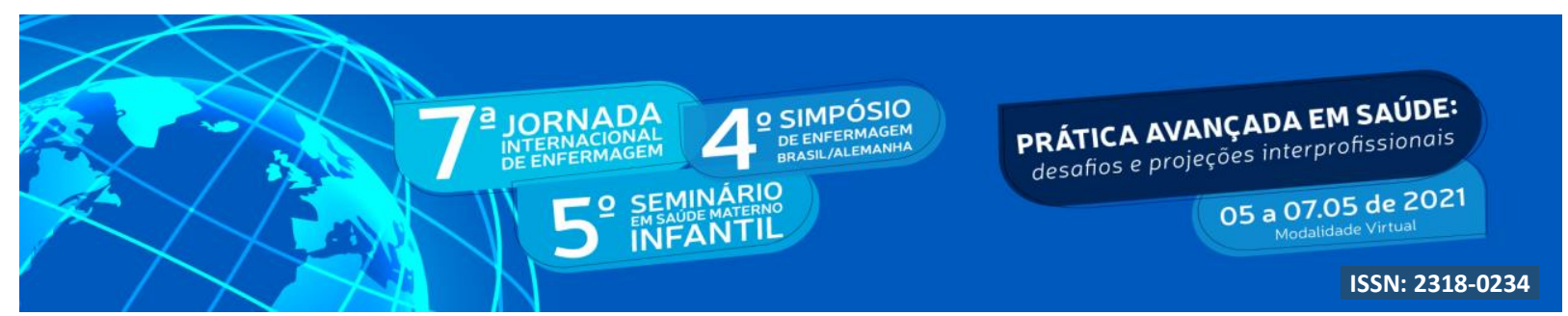

biológicas/clínica; Observação; outros tipos (não foi possível definir).

Em vista de corresponder aos objetivos da pesquisa, foram aplicados critérios de inclusão e de exclusão para o trato do material. Os critérios de inclusão foram: projetos originais e escritos na língua portuguesa; projetos de pesquisa a serem realizados na APS; projetos que obtivessem todas as informações completas, referentes às categorias utilizadas para a pesquisa; projetos de pesquisa a serem realizados no município de Santa Maria. Como critérios de exclusão foram considerados: projetos duplicados; projetos que estivessem com informações ausentes de categorias utilizadas para a pesquisa; projetos de pesquisa a serem realizados em locais que não incluíam a APS; projetos de pesquisa a serem realizados fora do município de Santa Maria.

\section{RESULTADOS E DISCUSSÃO}

Assim, foram encontrados sete projetos de pesquisa do ano de 2014; 76 do ano de 2015; 81 do ano de 2016; 36 do ano de 2017; 67 do ano de 2018; 88 do ano de 2019 e 67 do ano de 2020, totalizando 422 projetos de pesquisa. Com a aplicação dos critérios de inclusão e de exclusão, resultou 1 projeto do ano de 2014; 31 do ano de 2015; 37 do ano de 2016; 21 do ano de 2017; 39 do ano de 2018; 37 do ano de 2019 e 29 do ano de 2020.

Foram analisadas nesta pesquisa 195 projetos de pesquisas, dentre os anos de 2014 a 2020, armazenadas no NEPeS, sendo possível obter uma caracterização do perfil de pesquisas na APS no município de Santa Maria. No que tange a categoria local de realização se observou que durante estes determinados anos as pesquisas se realizaram em sua maioria nas UBS 64 (33\%), seguido da ESF, 54 (28\%), UBS e ESF (mista), 45 (23\%), RAS 28 (14\%) e no Sistema de Gestão 4 (2\%).

O predomínio de pesquisas realizadas nas UBS pode ocorrer pelo fato que estas se constituem como um espaço efetivo para a qualificação em busca de melhor organização dos serviços e maior oferta de ações aos usuários (CAVALCANTI; OLIVEIRA NETO; SOUSA, 2015). Contudo, o Ministério da Saúde vem incentivando, nas últimas décadas, ações que possam promover a integralidade como uma proposta estruturante para melhorar as práticas de cuidado e educação desenvolvidas no SUS, dentre essas ações, encontra-se a ESF. A ESF 


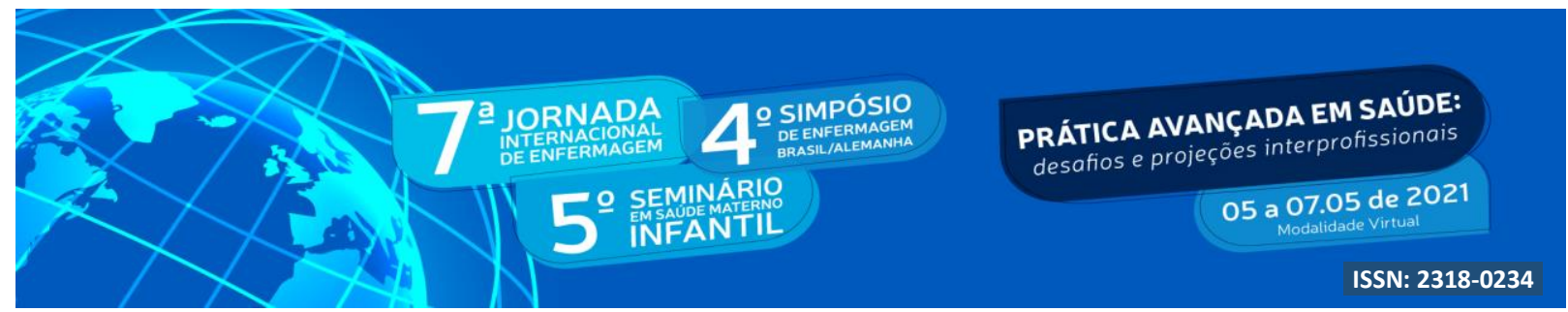

individuais, familiares e coletivas que visam a promoção, prevenção, proteção, tratamento, redução de danos, vigilância em saúde, entre outras ações (BRASIL, 2017b).

A análise das pesquisas aponta que durante os anos que conpuseram a amostra desta pesquisa se obteve a prevalência na categoria de participantes os usuários do serviço 115 (49\%), seguido dos profissionais 87 (37\%), análise documental $13(6 \%)$, alunos 10 (4\%), gestores $4(2 \%)$, professores $3(1 \%)$ e outros $1(0 \%)$.

Conforme a análise dos dados se observa o predomínio de usuários participantes de pesquisas na APS, na literatura se encontra que investigar e discutir a opinião de quem é cuidado, acerca da assistência recebida, pode ser uma potente ferramenta para garantir a qualidade da atenção (SENA et al, 2015). Com esse achado percebe-se que a escuta da opinião dos usuários pode apresentar melhorias nos serviços de saúde, tendo em vista que assim se compreende as necessidades dos usuários planejando ações direcionadas, tornando o cuidado resolutivo e integral.

Observa-se ainda a falta de retorno -“feedback"- para os participantes das pesquisas, de um lado como fator de desmotivação, e de outro, como impedimento da reflexão dos processos de práticas em saúde e demais assuntos que as pesquisas possam abordar na APS. É importante que as pesquisas realizadas na APS não sejam meras pesquisas engavetadas e sem aproveitamento após sua realização, e sim, que sirvam com o objetivo final de potencialização das práticas em saúde de um determinado serviço de saúde (SANTOS; BARROS; DELDUQUE, 2019).

Em relação ao instrumento de coleta se obeteve predomínio da entrevista 91 (42\%), seguindo do questionário 82 (38\%), análise documental/prontuário 23 (11\%), abordagem grupal $9(4 \%)$, outros $6(3 \%)$, observação $4(2 \%)$ e por útimo análise biológicas/clínicas 2 $(1 \%)$.

De acordo com esta caracterização se percebe que a maioria das pesquisas utilizaram como instrumento de coleta a entrevista e o questionário, estes instrumentos são em sua maioria utilizados para se obter informações subjetivas e objetivas dos participantes das pesquisas, sendo ferramentas importantes para validação das pesquisas realizadas na APS.

A entrevista é realizada através de coleta de dados sobre um determinado fenômeno e é a técnica mais utilizada no processo de trabalho de campo. Por meio dela os pesquisadores 


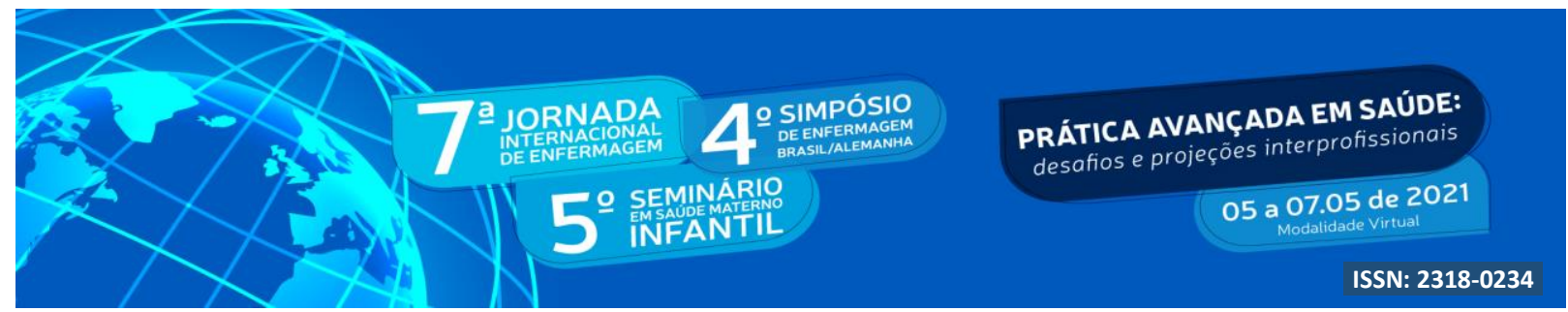

buscam coletar dados objetivos e subjetivos. Considera-se a entrevista como uma modalidade de interação entre duas ou mais pessoas, possibilitando que o entrevistado expresse seus reais sentimentos de acordo com a temática a ser discutida, visto que a entrevista possibilita uma gama de ideias, e por vezes as perguntas podem ser mais complexas, e para isso o facilitador, -neste caso o entrevistador,- estará presente para sanar quaisquer dúvidas referente a entrevista (BATISTA; MATOS; NASCIMENTO, 2017).

Uma pesquisa científica requer um longo planejamento, disponibilidade de recursos humanos e materiais, tempo e empenho. A aplicabilidade de instrumentos, como por exemplo questionários e formulários, é uma forma efetiva para o levantamento de dados populacionais com relativa facilidade administrativa. Com um planejamento adequado dos formulários utilizados em pesquisas em saúde, se tem um menor índice de vieses e erros na execução do estudo científico (THOMAS; OENNING; GOULART, 2018).

\section{CONCLUSÃO}

O estudo mostrou que as pesquisas realizadas na APS são em sua maioria realizadas nas Unidades Básicas de Saúde, tendo como participantes majoritoriamente os usuários e utilizando como instrumento de coleta a entrevista. A caracterização das pesquisas é importante pois além de demostrar quais conteúdos e problemas de saúde estão sendo investigados, pode apresentar resultados que tragam beneficios aos serviços de saúde e usuários além de incentivar novos estudos.

Conclui-se que a realização de pesquisas no âmbito da APS serve para desenvolver e subsidiar tomadas de decisão baseadas em evidências científicas, podendo produzir ferramentas e meios estratégicos de investigação, qualificando o cuidado prestado e os processos de trabalho em saúde. A caracterização das pesquisas realizadas neste artigo, nos mostra que a integração ensino serviço comunidade a cada ano se fortalece, assim os problemas da APS são elencados e investigados com base em uma metodologia participativa envolvendo todos os sujeitos do cuidado.

\section{REFERÊNCIAS}




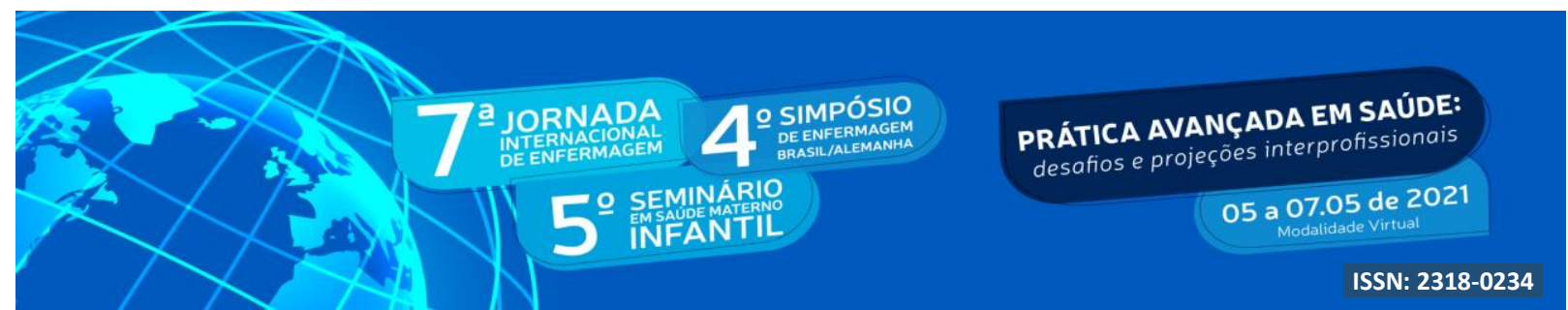

BATISTA, E. C.; MATOS, NASCIMENTO, A. B. A entrevista como técnica de investigação na pesquisa qualitativa. Revista Interdisciplinar Científica Aplicada. v.11, n.3, p.23-38, 2017.

BRASIL. Conselho Nacional de Saúde. Resolução n 569, de 8 de dezembro de 2017. Brasília, 2017a.

BRASIL. Ministério da Saúde. Portaria nº 2.436, de 21 de setembro de 2017. Aprova a Política Nacional de Atenção Básica, estabelecendo a revisão de diretrizes para a organização da Atenção Básica, no âmbito do Sistema Único de Saúde (SUS). Brasília, DF: Ministério da Saúde, 2017b.

BRASIL. Ministério da Saúde. Secretaria de Atenção à Saúde. Departamento de Atenção Básica. Política Nacional de Atenção Básica. Brasília, DF: Ministério da Saúde, 2012.

BRITO, M.C.C. et al. Formação do enfermeiro para a atenção básica: um olhar sobre o conhecimento produzido. SANARE, Sobral, v. 16, n. 2, p. 93-102, jul./dez. 2017.

CAMARA, A. M. C. S.; GROSSEMAN, S.; PINHO, D. L. M. Interprofessional education in the PET-Health Program: perception of tutors. Interface, Botucatu, v. 19, n. 1, p. 817-829, 2015.

CAVALCANTI, P. C. S.; OLIVEIRA NETO, A. V.; SOUSA, M. F. Quais são os desafios para a qualificação da Atenção Básica na visão dos gestores municipais?. Saúde em Debate, Rio de Janeiro, v. 39, n. 105, p.323-336, abr./jun. 2015.

CODATO, L.A.B. et al. Significados do estágio em Unidades Básicas de Saúde para estudantes de graduação. Revista da Abeno, v. 19, n. 1, p. 2-9, 2019.

PMSM/SMS - PREFEITURA MUNICIPAL DE SANTA MARIA. SECRETARIA MUNICIPAL DE SAÚDE. Plano Municipal de Saúde: 2018-2021. Santa Maria: SMS/Prefeitura de Santa Maria, 2018.

MORAIS, J.B. et al. Avaliação das pesquisas nos cenários da atenção primária à saúde: produção, disseminação e utilização dos resultados. Saúde Soc. São Paulo, v.27, n.3, p.783793, 2018.

SANTOS, A. DE O.; BARROS, F. P. C. DE; DELDUQUE, M. C. A pesquisa em saúde no Brasil: desafios a enfrentar. Saúde em Debate, v. 43, n. spe 5, p. 126-136, 2019.

SENA, A. L. C. et al. Acolhimento e satisfação do usuário na estratégia de saúde da família: uma experiência de êxito. Revista APS, v. 18, n. 2, p. 134-40, abr./jun., 2015.

THOMAS, D.B.; OENNING, N. S. X.; GOULART, B. N. G. Aspectos essenciais na construção de instrumentos de coleta de dados em pesquisas primárias de saúde. Rev. 


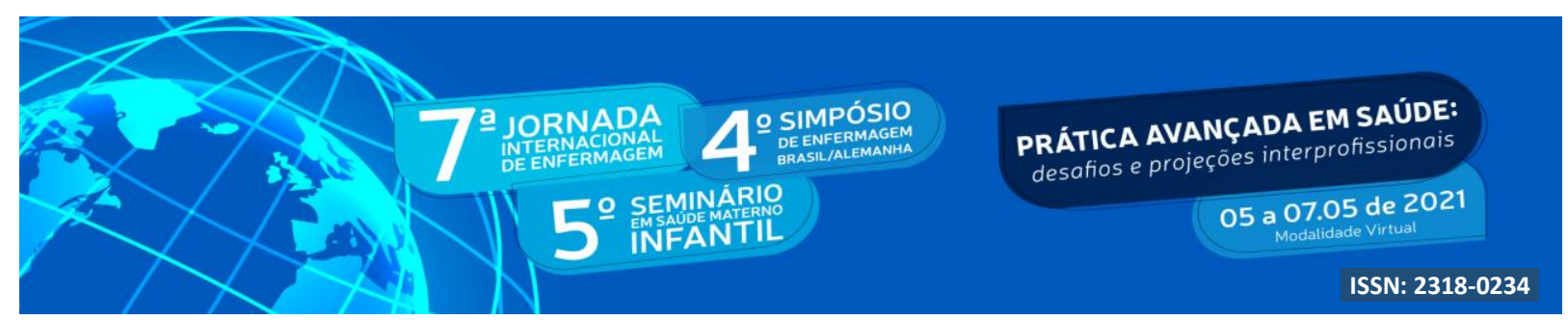

CEFAC. v. 20, n. 5, p. 657-664, set-out., 2018.

ZANELLA, L.G..C.H. Metodologia de Pesquisa. 2. ed. Florianópolis: Departamento de ciências da administração, UFSC, 2013. 